Revista peruana de biología 26(2): 227 - 234 (2019) doi: http://dx.doi.org/10.15381/rpb.v26i2.16378 ISSN-L 1561-0837; eISSN: 1727-9933

Universidad Nacional Mayor de San Marcos

\section{Efecto de la densidad de siembra en el desarrollo vegetativo del aguaje (Mauritia flexuosa L.f.) en plantaciones forestales}

\section{TRABAJOS ORIGINALES}

$\begin{array}{ll}\text { Presentado: } & 23 / 08 / 2018 \\ \text { Aceptado: } & 30 / 04 / 2019 \\ \text { Publicado online: } & 06 / 07 / 2019\end{array}$

\section{Correspondencia:}

* Autor para correspondencia

Luis Freitas Alvarado: Ifreitas@iiap.org.pe

Ricardo Zárate Gómez: rzarate@iiap.org.pe

Ricardo Bardales L.: rbardaleslozano@gmail.com

Dennis Del Castillo Torres: ddelcastillo@iiap.org.pe

Otros datos de los autores / biografía:

ORCID Luis Freitas: 0000-0002-3357-3476

Luis Freitas Alvarado, Programa de Investigación en Manejo Integral del Bosque y Servicios Ambientales (PROBOSQUES).

Ricardo Zárate Gómez, Programa de Investigación en Cambio Climático Desarrollo Territorial y Ambiente (PROTERRA)

Dennis Del Castillo Torres, Programa de Investigación en Manejo Integral del Bosque y Servicios Ambientales (PROBOSQUES).

Citación:

Freitas Alvarado L., R. Zárate Gómez, R. Bardales Lozano y D. Castillo Torres. 2019. Efecto de la densidad de siembra en el desarrollo vegetativo del aguaje (Mauritia flexuosa L.f.) en plantaciones forestales. Revista peruana de biología 26(2): 227 - 234 (Julio 2019). doi: http://dx.doi.org/10.15381/ rpb.v26i2.16378

Palabras clave: Aguaje; crecimiento de palmeras; reforestación; conservación; silvicultura; productos forestales no maderables.

Keywords: Aguaje; Palm growth; reforestation; conservation; silviculture; non-timber forest products.

\section{Effect of sowing density on the vegetative development of aguaje (Mauritia flexuosa L.f.) in forest plantations}

\author{
Luis Freitas Alvarado ${ }^{1 *}$, Ricardo Zárate Gómez ${ }^{1}$, Ricardo Bardales \\ Lozano $^{2}$ y Dennis Del Castillo Torres ${ }^{1}$ \\ 1. Instituto de Investigaciones de la Amazonía Peruana (IIAP). Av. Quiñones km 2.5, San \\ Juan Bautista, Maynas, Loreto, Perú. \\ 2. Palmas del Espino S.A., Departamento de Investigación y Desarrollo Agrícola. Pal- \\ mawasi S/N-Uchiza, Tocache, San Martín, Perú.
}

\section{Resumen}

En este trabajo es estudiado el efecto de dos densidades de siembra de la palmera aguaje (Mauritia flexuosa L.f.) sobre su crecimiento en altura; cuantificamos la proporción de plantas según sexo y determinamos los requerimientos ecológicos de iluminación y forma de copa del aguaje. El estudio se desarrolló en dos subparcelas con diferente densidad de siembra que fueron monitoreadas por 6 años después de 6 años de haber sido plantadas. Los resultados muestran que los individuos presentan mayor altura promedio y por lo tanto mayor crecimiento a mayor densidad de siembra. Es así que a los 12 años de establecidas las plantaciones, los individuos tuvieron en promedio $15.28 \mathrm{~m}$ y $13.1 \mathrm{~m}$ de altura a densidades de siembra mayores y menores, respectivamente. La proporción de palmeras fértiles se fue incrementando con el tiempo, siendo por lo general mayor a bajas densidades e incrementando en el tiempo la presencia de plantas femeninas. Existe una marcada competencia entre las copas de las palmeras por requerimientos de luz en las categorías buena y aceptable, por lo que se recomienda continuar con la evaluación y analizar el comportamiento futuro de las palmeras.

\section{Abstract}

In this work, the effect of two planting distances of Mauritia flexuosa on its height is studied, quantified the sex ratio and ecological requirements needed by the species, e.g. light and crown shape. We conducted this study in two plots, each one with a different planting distance, which were monitored throughout 6 years starting after 6 years of the establishment of the plantations. Increased planting distance in between individuals gave increased average height and thus increased growth. Individuals planted with higher vs lower distances grew in average $15.28 \mathrm{~m}$ and 13.1 $m$ in height respectively. The proportion of fertile palms increased throughout time and was lower in plantations with higher palm densities. The presence of female individuals increased also with time. There was a strong competition among palm crowns related to light requirements in good and acceptable categories. We recommended to continue with the evaluation and future analysis of this species' behavior. 


\section{Introducción}

Mauritia flexuosa L.f. es una palmera de gran importancia social, económica y ambiental en la Amazonia peruana. En el Perú, se le conoce con el nombre de "aguaje", en Colombia como "moriche" y en el Brasil "buriti" (Del Castillo et al. 2010). Esta especie es nativa de la selva amazónica y tiene una amplia distribución en América del Sur (Henderson 1995). Es una especie dioica, es decir, presenta las inflorescencias separadas en individuos masculinos y femeninos. Los individuos adultos alcanzan hasta $35 \mathrm{~m}$ de altura y de 23-50 (-80) cm de diámetro (Henderson 1995).

En su ambiente natural, M. flexuosa crece en zonas inundables en extensas formaciones monoespecíficas, "aguajales". Sin embargo, también crece bien en suelos de buen drenaje y se considera que la especie es apta para ser cultivada en diversas condiciones de suelo en la selva baja. La edad reproductiva se inicia a los 8 ó 10 años de edad (Flores 1997), la producción de frutos es estacional, así, en la zona de Iquitos la mayor producción coincide con la época lluviosa de diciembre a mayo (Díaz et al. 2015). El principal producto aprovechable son los frutos, con altos valores de vitamina A, alrededor de 30 mg por $100 \mathrm{~g}$ de pulpa (Santos 2005), los que son consumidos en forma directa como frutos maduros, en refrescos (aguajina), masa de aguaje, helados, chupetes (Rojas 2001). Por otro lado, los suelos de los ecosistemas de aguajal constituyen importantes sumideros de carbono y contienen entre 532 - 632 t/ha (Guzmán 2004).

La alta demanda de frutos de aguaje en las ciudades amazónicas viene afectando a los "aguajales" debido a que la cosecha de los frutos se realiza de manera destructiva. Se calcula que la demanda de aguaje por la ciudad de Iquitos es de aproximadamente 21.9 t por día, lo que implica que diariamente se cortan unas 12000 palmeras hembras de su medio natural (García 2001, Del Castillo et al. 2010). Por lo tanto, existe una necesidad de generar alternativas para la cosecha sostenible de los frutos de esta palmera.

Una de las opciones para frenar la presión sobre las poblaciones naturales de aguaje es el establecimiento de plantaciones con selección de características comerciales específicas de calidad de frutos, orientadas a solucionar diversos problemas en el aspecto productivo, ambiental y social. Las plantaciones forestales constituyen una forma de incrementar el abastecimiento de materia prima para la industria, recuperar áreas deforestadas con nueva cobertura vegetal, obtener una mayor captación de agua y mejorar los servicios ambientales de almacenamiento y secuestro de carbono. Además, las plantaciones generan ingresos a los productores, los cuales manejan áreas cercanas a sus viviendas, minimizando distancias para la cosecha de los frutos, y promoviendo así el desarrollo agroindustrial de la región (Delgado et al. 2007, Horn et al. 2012).

No se cuenta con registros y se desconoce la superficie de plantaciones de aguaje en la Amazonía peruana; sin embargo, hay evidencias de la existencia de sistemas productivos asociados al cultivo del aguaje en predios familiares de comunidades amazónicas. Por ejemplo, en comunidades de la cuenca del río Marañón se ha reportado el cultivo del aguaje en plantaciones puras o asociadas con otros frutales (Bejarano \& Pianna 2002, Manzi \& Coomes 2009). Así mismo, es muy poco lo que se ha avanzado sobre la silvicultura y manejo de plantaciones de aguaje, y no se cuenta con los conocimientos suficientes para el establecimiento y manejo de esta importante palmera. Por lo tanto, existen vacíos de información sobre las técnicas para la implementación de un programa que nos permita establecer plantaciones a escala comercial en la Amazonía.

El Programa de Investigación de Manejo de Bosques y Servicios Ambientales del Instituto de Investigaciones de la Amazonía Peruana (IIAP) tiene una larga trayectoria en el conocimiento de la ecología de los aguajales en su ecosistema. Desde el 2004, el IIAP viene promocionando el mejoramiento genético de la especie con el fin de fomentar la selección de genotipos precoces en la producción de frutos (IIAP-INCAGRO 2010). En el presente estudio, hemos probado el efecto de dos tratamientos de densidad de siembra en el desarrollo vegetativo del aguaje en plantaciones forestales. Asimismo, ponemos énfasis en la evaluación de la fenología y las condiciones silviculturales como requerimientos de iluminación y forma de copa del aguaje en plantaciones.

\section{Material y métodos}

Área de Estudio.- Las plantaciones experimentales de aguaje fueron establecidas en el Centro de Investigaciones Jenaro Herrera del IIAP, ubicada a $2.7 \mathrm{~km}$ de Villa Jenaro Herrera, a $200 \mathrm{~km}$ aguas arriba de la ciudad de Iquitos. La zona de estudio pertenece al distrito de Jenaro Herrera, provincia de Requena, departamento de Loreto, Perú; y se localiza en las coordenadas: $4^{\circ} 54$ ' 11.66" S; $73^{\circ} 40^{\prime} 8.976 " \mathrm{~W}$, a $125 \mathrm{~m}$ de altitud (Figura 1).

La temperatura mensual promedio de la zona es de $26.4{ }^{\circ} \mathrm{C}$ con pequeñas fluctuaciones durante el año y la temperatura diaria promedio es de $29.9^{\circ} \mathrm{C}$ (Baluarte, 2012). Las temperaturas absolutas más elevadas se registran de enero a marzo y de agosto a octubre; las más bajas generalmente ocurren en julio.

La precipitación media anual es de $2730 \mathrm{~mm}$, con lluvias constantes durante casi todos los meses del año. Abril es el mes de mayor precipitación con $294.3 \mathrm{~mm}$ y julio el de menor llegando a $155.2 \mathrm{~mm}$. El período más lluvioso comprende los meses de octubre a mayo y el período de menor precipitación comprende los meses de junio a setiembre (Baluarte 2012). La estacionalidad pluviométrica, aunque poco marcada, tiene gran importancia en los fenómenos que provocan la florescencia. El $80 \%$ de las especies del bosque de altura en la zona de Jenaro Herrera presentan ritmos de floración correlacionadas con las precipitaciones y dispersan sus frutos siempre una vez al año entre diciembre y abril (Gauthier \& Spichiger 1986).

Las plantaciones han sido instaladas sobre suelos de color amarillo anaranjado (acrisol órtico) de las llanuras y laderas de la terraza alta, fuertemente ácidos, con una 


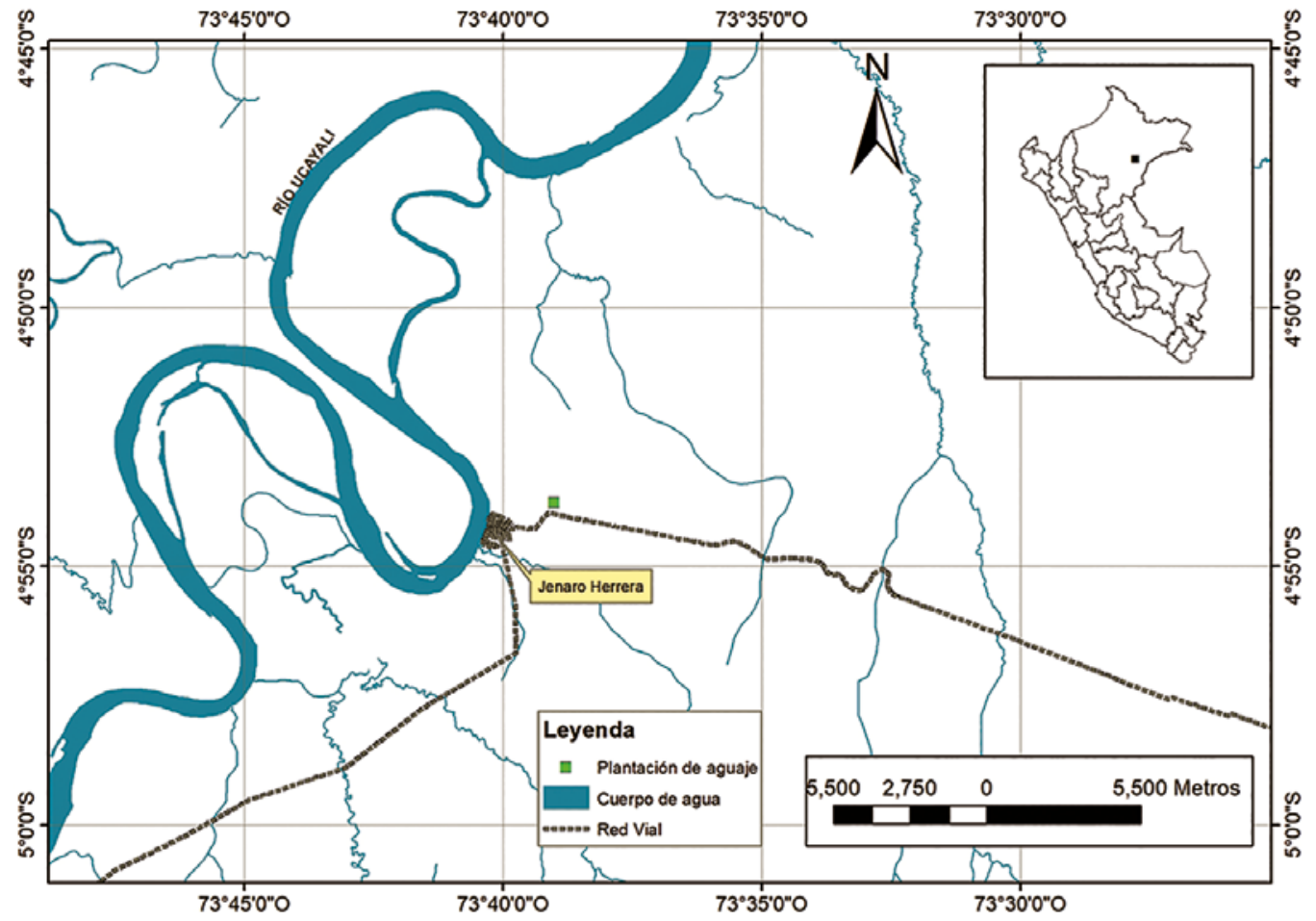

Figura 1. Mapa de ubicación de las plantaciones de Mauritia flexuosa (aguaje), cercano a Jenaro Herrera, en la cuenca del rio Ucayali, departamento de Loreto, Perú.

textura que va de franco areno arcilloso a arcillo arenoso sin fragmentos gruesos y de color pardo oscuro. Los suelos presentan una estructura granular con intensa presencia de raíces hasta una profundidad de $60-70$ $\mathrm{cm}$, por debajo de la cual la estructura se transforma en masiva. Algunos suelos presentan un horizonte superior grisáceo bien desarrollado (Claussi et al. 1992).

Diseño de la Investigación.- En diciembre del 2002, se estableció una plantación forestal de aguaje de 5000 $\mathrm{m}^{2}$ dividida en dos subparcelas de $2500 \mathrm{~m}^{2}$ cada una. La plantación se generó a partir de semilla botánica obtenida de una palmera madre de porte bajo (estípite menor de $2.5 \mathrm{~m}$ de altura) ubicada en la comunidad de "Varillal" en la zona de la carretera Iquitos-Nauta, en las coordenadas: $3^{\circ} 52^{\prime} 48.413^{\prime \prime}$ S; $73^{\circ} 20^{\prime} 49.69^{\prime \prime}$ W. Las subparcelas presentan diferentes densidades de siembra, la primera con una densidad de $8 \mathrm{~m}$ entre plantas y una planta en el centro ("quinconcé") con un total de 85 plantas. En esta parcela, se marcaron 15 plantas ubicadas en el centro de la subparcela para la evaluación de las variables biométricas. La segunda subparcela fue establecida con densidades entre plantas de $7 \mathrm{~m}$ (diseño de plantación tipo "marco real") con un total de 52 plantas (Figura 2). En esta segunda subparcela, se marcaron 29 plantas para la evaluación de las variables biométricas.

La plantación fue establecida a campo abierto en un área de bosque secundario de aproximadamente 20 años de edad, donde inicialmente hubo un bosque primario que luego fue desboscado para la instalación de diferentes sistemas productivos; posteriormente la parcela fue abandonada.
Información registrada.- Se realizaron mediciones biométricas de las estructuras vegetativas e inflorescencias de las palmeras marcadas en cada subparcela a partir de los seis años de establecida la plantación y durante los seis años de duración del estudio. Los caracteres cuantitativos tomados están basados en los descriptores para el aguaje publicado por Freitas et al. (2006), tales como: altura total, número de hojas vivas, longitud del peciolo, diámetro del peciolo, número de foliolos, número de racimos, longitud del pedúnculo y raquis del racimo. Los valores promedios de los caracteres cuantitativos fueron comparados entre las dos densidades de siembra, para ello se hizo el t-test después de corroborar una distribución normal de datos y similar variabilidad entre parcelas.

Así mismo, se hizo un monitoreo fenológico anual de la floración a todos los individuos de las subparcelas

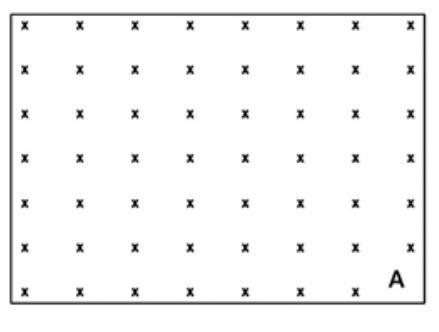

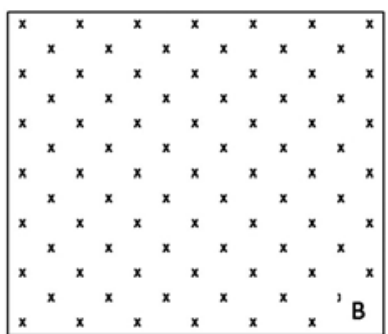

Figura 2. Sistemas de plantación establecidas para el estudio en Mauritia flexuosa (aguaje). (A) Marco real, con distanciamiento de 7 m entre plantas, y (B) Quinconce, con distanciamiento de $8 \mathrm{~m}$ entre plantas formando un cuadrado y una planta en el centro. 
para determinar la fenología de las palmeras a diferentes edades. Consideramos a una palmera adulta cuando el individuo desarrolló uno o más racimos con flores y/o frutos; en este caso, se diferenció el sexo de las flores en masculinas y femeninas. Cuando las palmeras no registraron la presencia de flores y/o frutos, las consideramos como subadultas.

La condición silvicultural se registró en todas las palmeras de las dos subparcelas, midiendo dos variables cuantitativas: la iluminación de la copa y forma de la copa, aplicando la metodología usada por Hutchinson (1993) y Synnott (1991). Iluminación de la copa: 1- Muy buena, copa completamente libre, recibe luz directa vertical y horizontalmente; 2- Buena, copa completamente libre hacia arriba, solo recibe luz directa verticalmente 3- Aceptable, copa parcialmente libre hacia arriba, recibe poca luz directa verticalmente; 4- Deficiente, copa parcialmente cubierta, recibe luz directa lateral; y 5- Muy deficiente, copa completamente cubierta, no recibe luz directa. Forma de copa: 1- Copa de forma perfecta, círculo completo; 2- Copa de forma buena, círculo irregular; 3- Copa de forma aceptable, media copa; 4- Copa de forma pobre, menos de media copa y 5 - Copa de forma muy pobre, menos de cinco hojas.

Procesamiento de datos.- Los datos cuantitativos vegetativos y de la producción fueron evaluados mediante análisis descriptivo estimando el promedio y el error estándar de las observaciones anuales en cada parcela. Para una mejor ilustración comparativa del crecimiento de la planta entre los sistemas de plantación (Densidad de siembra) se ploteó mediante diagrama de cajas la altura de planta por cada año de evaluación.
Las variables de la fenología de la reproducción se evaluaron en base al número de individuos adultos observados con inflorescencia masculina y femenina $\mathrm{y}$ los subadultos que no entraron aún en producción, los cuales posteriormente fueron expresados en porcentaje para cada parcela evaluada anualmente. Así mismo, se calculó el porcentaje de iluminación y la forma de la copa para cada parcela de evaluación desagregados en 5 categorías: iluminación de copa (Hutchinson, 1993): 1- Muy buena, 2- Buena, 3- Aceptable, 4- Deficiente y 5- Muy deficiente); y forma de copa Synnott (1991): 1- Perfecta, 2Buena, 3- Aceptable, 4- Pobre y 5- Muy pobre.

\section{Resultados y discusión}

Crecimiento de las plantas.- En la Figura 3 se presenta el diagrama de cajas con información sobre el crecimiento en altura entre los 6 y 12 años de instalada la plantación y según densidad de siembra. La serie histórica de los datos de crecimiento presenta un incremento continuo con la edad de la plantación. Si bien es cierto que los promedios de alturas de la plantación con mayor densidad de siembra presenta mayores alturas, la figura muestra que no existen diferencias estadísticas significativas entre los promedios de altura de las dos subparcelas según densidad de siembra.

De igual forma, tal como se muestra en la Tabla 1, la altura promedio anual de la parcela con mayor densidad de siembra es superior. Así, a los 6 y 12 años es de $9.09 \pm 0.27 \mathrm{~m}$ y $15.28 \pm 0.36 \mathrm{~m}$, respectivamente, mientras que la de menor densidad presenta valores de $7.72 \pm 0.32$ y $13.15 \pm 0.35 \mathrm{~m}$. Otros caracteres vegetativos como el número de hojas, número de foliolos, longitud y diámetro

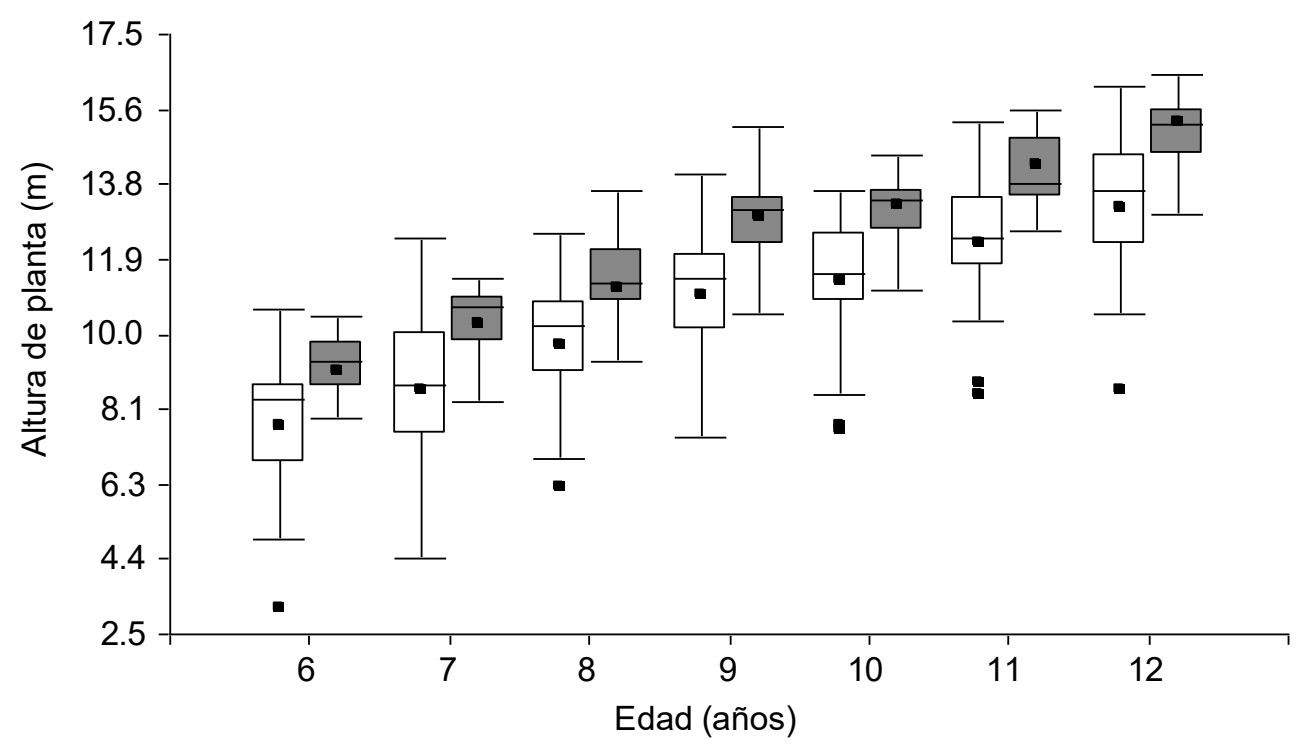

Marco real $\square$ Quinconcé

Figura 3. Diagrama de cajas de la relación entre edad y altura de Mauritia flexuosa L.f. "aguaje" en plantaciones forestales con diferente densidad de siembra. 
del peciolo, también muestran un crecimiento continuo con la edad de la plantación (Tabla 1).

La altura promedio de las palmeras en las dos subparcelas a la edad de 6 años de establecida la plantación son superiores a los registrados por Rojas (2000), quien reporta alturas promedio de $5.1 \mathrm{~m}$ en una plantación de la misma edad con un distanciamiento entre plantas de $6 \times 6 \mathrm{~m}$. Consideramos que una de las razones para un mayor crecimiento en altura de la plantación con mayor densidad de siembra, $8 \mathrm{~m}$ y uno en el centro, podría estar determinado por una mayor competencia lumínica entre plantas. Mauritia flexuosa L.f. está considerada como una especie heliófita (Cárdenas Valencia, 1985). Gonzáles-B. y Rial (2011) indican que la ausencia de luz limita el crecimiento de los juveniles de la especie en su medio natural. Sin embargo existen otros factores adicionales que influyen en el crecimiento de las plantas, como tipo de suelo, fisiografía, factores climatológicos y otros (Villasana \& Suárez 1997, Dambroth \& Bassam 1983), factores genéticos (Rathcke \& Lacey 1985, Alencar 1994)

En la Tabla 2 se presentan los caracteres cuantitativos de las inflorescencias de individuos adultos. Existe una tendencia de que, a mayor edad de la plantación, se incrementa el número de individuos adultos fértiles. En ese sentido, Urrego (1987) manifiesta que los factores climáticos son los que probablemente tienen mayor influencia en la floración, entre ellos el brillo solar y la diferencia entre temperaturas extremas.
Los otros caracteres de la inflorescencia, como número de racimos, longitud del pedúnculo y longitud del raquis, presentan variaciones marcadas en los diferentes años del estudio. Los valores son similares a los presentados por Villachica (1996), quien reporta longitudes del pedúnculo que varían entre 0.6 y $1 \mathrm{~m}$ y para el raquis un promedio de $1.86 \mathrm{~m}$, variando entre 1.54 a $2.8 \mathrm{~m}$; mientras que Gonzales (2006) presenta promedios de $0.92 \mathrm{~m}$ para el pedúnculo y $1.88 \mathrm{~m}$ para el raquis.

Fenología del aguaje.- En la Tabla 3 se muestra el porcentaje de individuos de la plantación que alcanzó la madurez sexual durante los seis años de evaluación. Es importante notar que, a pesar que la tendencia fue creciente en el porcentaje de individuos adultos en el tiempo, los valores más altos fueron reportados en la subparcela con menor densidad de siembra (marco real $=52$ individuos), llegando a tener $61.5 \%$ de los individuos plantados en condición de adultos fértiles a los 12 años, mientras que la otra subparcela alcanzó un $42.4 \%$. En cuanto al porcentaje de individuos femeninos, observamos que la plantación con menor densidad de siembra, marco real (52 individuos), presenta $28.8 \%$ de individuos femeninos, mientras que el método con mayor densidad de siembra (85 individuos), denominado quinconcé, solo obtuvo un $22.4 \%$ de individuos femeninos.

La distribución porcentual según sexo de las palmeras de aguaje adultas en una plantación es de gran importan-

Tabla 1. Valores promedios ( \pm error estándar) de los caracteres cuantitativos vegetativos evaluados a plan|tas de Mauritia flexuosa L.f (aguaje) en plantaciones forestales con diferente densidad de siembra.

\begin{tabular}{|c|c|c|c|c|c|c|c|c|}
\hline \multirow{2}{*}{$\begin{array}{l}\text { Edad de } \\
\text { plantación } \\
\text { (años) }\end{array}$} & \multicolumn{4}{|c|}{ Quinconcé ( $n=15$ ind.) } & \multicolumn{4}{|c|}{ Marco Real ( $n=29$ ind.) } \\
\hline & $\begin{array}{l}\text { Número } \\
\text { hojas }\end{array}$ & $\begin{array}{l}\text { Longitud } \\
\text { peciolo }\end{array}$ & $\begin{array}{l}\text { Diámetro } \\
\text { peciolo }\end{array}$ & $\begin{array}{c}\text { Altura } \\
\text { total }\end{array}$ & $\begin{array}{l}\text { Número } \\
\text { hojas }\end{array}$ & $\begin{array}{l}\text { Longitud } \\
\text { peciolo }\end{array}$ & $\begin{array}{l}\text { Diámetro } \\
\text { peciolo }\end{array}$ & $\begin{array}{l}\text { Altura } \\
\text { total }\end{array}$ \\
\hline 6 & $8.60 \pm 0.38$ & $4.26 \pm 0.15$ & $6.47 \pm 0.12$ & $9.09 \pm 0.27$ & $8.04 \pm 0.36$ & $3.63 \pm 0.17$ & $4.89 \pm 0.53$ & $7.72 \pm 0.32$ \\
\hline 7 & $8.73 \pm 0.54$ & $4.30 \pm 0.17$ & $6.57 \pm 0.23$ & $10.27 \pm 0.32$ & $8.46 \pm 0.35$ & $3.90 \pm 0.11$ & $6.40 \pm 0.09$ & $8.63 \pm 0.37$ \\
\hline 8 & $8.93 \pm 0.60$ & $4.51 \pm 0.16$ & $6.85 \pm 0.20$ & $11.16 \pm 0.44$ & $8.77 \pm 0.37$ & $4.09 \pm 0.16$ & $7.11 \pm 0.11$ & $9.72 \pm 0.29$ \\
\hline 9 & $9.00 \pm 0.58$ & $4.70 \pm 0.17$ & $7.04 \pm 0.32$ & $12.96 \pm 0.28$ & $8.81 \pm 0.42$ & $4.11 \pm 0.10$ & $7.17 \pm 0.10$ & $11.01 \pm 0.29$ \\
\hline 11 & $9.00 \pm 0.50$ & $4.80 \pm 0.12$ & $7.57 \pm 0.23$ & $14.25 \pm 0.41$ & $9.69 \pm 0.41$ & $4.16 \pm 0.08$ & $7.32 \pm 0.13$ & $12.28 \pm 0.29$ \\
\hline 12 & $9.50 \pm 0.44$ & $4.91 \pm 0.13$ & $7.58 \pm 0.24$ & $15.28 \pm 0.36$ & $9.81 \pm 0.39$ & $4.51 \pm 0.08$ & $7.84 \pm 0.12$ & $13.15 \pm 0.35$ \\
\hline
\end{tabular}

Tabla 2. Valores promedios ( \pm error estándar) de los caracteres cuantitativos de inflorescencias de plantas de Mauritia flexuosa L.f (aguaje) en plantaciones forestales con diferente densidad de siembra.

\begin{tabular}{|c|c|c|c|c|c|c|c|c|}
\hline \multirow{2}{*}{$\begin{array}{c}\text { Edad de } \\
\text { plantación } \\
\text { (años) }\end{array}$} & \multicolumn{4}{|c|}{ Quinconcé } & \multicolumn{4}{|c|}{ Marco Real } \\
\hline & $n^{*}$ & $\begin{array}{l}\text { Número } \\
\text { racimos }\end{array}$ & $\begin{array}{c}\text { Longitud } \\
\text { pedúnculo }\end{array}$ & $\begin{array}{l}\text { Longitud } \\
\text { raquis }\end{array}$ & $\mathrm{n}^{*}$ & $\begin{array}{l}\text { Número } \\
\text { racimos }\end{array}$ & $\begin{array}{c}\text { Longitud } \\
\text { pedúnculo }\end{array}$ & $\begin{array}{c}\text { Longitud } \\
\text { raquis }\end{array}$ \\
\hline 6 & 1 & $4.00 \pm 0.00$ & $0.38 \pm 0.00$ & $1.49 \pm 0.00$ & 3 & $3.00 \pm 1.15$ & $0.56 \pm 0.09$ & $1.57 \pm 0.13$ \\
\hline 7 & 7 & $2.80 \pm 0.58$ & $0.53 \pm 0.08$ & $1.54 \pm 0.21$ & 9 & $2.33 \pm 0.41$ & $0.69 \pm 0.09$ & $1.79 \pm 0.22$ \\
\hline 8 & 20 & $2.65 \pm 0.34$ & $0.84 \pm 0.04$ & $1.73 \pm 0.13$ & 27 & $3.33 \pm 0.32$ & $0.88 \pm 0.03$ & $1.78 \pm 0.07$ \\
\hline 9 & 8 & $1.43 \pm 0.30$ & $0.54 \pm 0.06$ & $1.41 \pm 0.10$ & 14 & $1.64 \pm 0.29$ & $0.75 \pm 0.04$ & $1.56 \pm 0.06$ \\
\hline 11 & 16 & $1.75 \pm 0.21$ & $0.67 \pm 0.05$ & $1.72 \pm 0.08$ & 15 & $2.47 \pm 0.40$ & $0.67 \pm 0.05$ & $1.75 \pm 0.07$ \\
\hline 12 & 36 & $2.64 \pm 0.19$ & $0.77 \pm 0.03$ & $1.68 \pm 0.07$ & 32 & $3.39 \pm 0.26$ & $0.72 \pm 0.03$ & $1.64 \pm 0.08$ \\
\hline
\end{tabular}

*Se evaluaron solo individuos adultos fértiles en ambas parcelas 
cia, teniendo en consideración que solamente las plantas femeninas son las que producen frutos. En condiciones naturales sin intervención (aguajales no aprovechados), la proporción de plantas masculinas y femeninas es de $51 \%$ y $49 \%$, respectivamente (Urrego 1987). Solo en aguajales con intensidad de aprovechamiento fuerte la relación porcentual de plantas según sexo varía, ya que se cortan los femeninos quedando una mayor reserva de individuos masculinos (82.64\%) y femeninos (17.36\%), de acuerdo a Freitas (2012) y Freitas \& Flores (2015).

Es interesante notar que nuestros resultados también muestran porcentajes muy similares de individuos adultos masculinos y femeninos a diferente densidad de siembra en la plantación a los 12 años de edad, comparados con aguajales no aprovechados (Tabla 3). A pesar que es de esperar que más individuos lleguen a la madurez sexual con el tiempo y más individuos femeninos serán productivos, la producción estará determinada por la producción de al menos el 50\% de individuos. Es decir, la proporción de individuos femeninos y masculinos debería ser de 50\% y 50\%. Desarrollar la tecnología para poder conocer el sexo a edad temprana de los aguajes sería muy útil para aumentar la producción de aguaje en plantaciones, reemplazando algunos individuos masculinos por nuevos plantones femeninos.

Existen pocas plantas que iniciaron la etapa reproductiva tempranamente, es decir, a los seis años de instalada la plantación. Sin embargo, estos individuos tienen gran importancia en el mejoramiento genético del aguaje debido a su precocidad en la producción de frutos. El aguaje en su medio natural inicia la floración entre los 8 y 10 años de edad (Flores 1997). Es interesante notar que estos individuos estuvieron presentes a diferentes intensidades de siembra dando alternativas viables para la fijación de este carácter en el programa de mejoramiento genético del IIAP.

Nuestros resultados permiten esclarecer ciertas dudas arraigadas entre los agricultores de la región, quienes comentan que en las plantaciones de aguaje existe una predominancia de palmeras masculinas. En el presente estudio mostramos que la proporción de sexos es similar al bosque natural y, por lo tanto, con la producción de frutos en plantaciones es posible conseguir una alta rentabilidad económica en forma sostenible. Por lo tanto, la necesidad de fomentar los estudios para conocer el sexo del aguaje a temprana edad podría también ayudar a fomentar el desarrollo de las plantaciones de aguaje en la región.

Condición silvicultural.- La condición silvicultural de las palmeras, de acuerdo a la cantidad de luz que reciben sus copas, muestra que la mayoría de los individuos se encuentran en condiciones de buena y aceptable (Tabla 4). El porcentaje de plantas es similar en las dos subparcelas: pocos individuos tienen iluminación de copa muy buena (7\%), existiendo un mayor porcentaje de individuos en la categoría de iluminación buena (57\%) y aceptable (35\%). El monitoreo de las plantaciones debe continuar debido a que las plantas aún se encuentran en crecimiento. La evaluación de la iluminación de copas nos ayudará más adelante a la aplicación de métodos de manejo silvicultural de las plantaciones eliminando las plantas que queden suprimidas. Debemos tener en cuenta que el aguaje es una palmera heliófita, es decir, requiere de abundante luz para crecer. Distan-

Tabla 3. Comparación del porcentaje de plantas de Mauritia flexuosa L.f (aguaje) según estado fenológico en plantaciones forestales con diferente densidad de siembra.

\begin{tabular}{|c|c|c|c|c|c|c|}
\hline \multirow{2}{*}{$\begin{array}{c}\text { Edad de } \\
\text { plantación } \\
\text { (años) }\end{array}$} & \multicolumn{3}{|c|}{ Quinconcé ( $n=85$ ind.) } & \multicolumn{3}{|c|}{ Marco Real ( $n=52$ ind.) } \\
\hline & Subadulto & Adulto femenino & Adulto masculino & Subadulto & Adulto femenino & Adulto masculino \\
\hline 6 & 98.8 & 0.0 & 1.2 & 94.2 & 1.9 & 3.8 \\
\hline 7 & 91.8 & 4.7 & 3.5 & 82.7 & 5.8 & 11.5 \\
\hline 8 & 76.5 & 8.2 & 15.3 & 48.1 & 25.0 & 26.9 \\
\hline 9 & 90.6 & 3.5 & 5.9 & 73.1 & 11.5 & 15.4 \\
\hline 11 & 81.2 & 5.9 & 12.9 & 71.2 & 11.5 & 17.3 \\
\hline 12 & 57.6 & 20.0 & 22.4 & 38.5 & 32.7 & 28.8 \\
\hline
\end{tabular}

\begin{tabular}{|c|c|c|c|c|}
\hline \multirow[b]{2}{*}{ Categoría } & \multicolumn{2}{|c|}{ Quinconcé ( $n=85$ ind.) } & \multicolumn{2}{|c|}{ Marco real ( $n=52$ ind.) } \\
\hline & Iluminación & Forma de copa & Iluminación & Forma de copa \\
\hline 1 & 7.1 & 0.0 & 7.7 & 0.0 \\
\hline 2 & 57.6 & 57.6 & 57.7 & 84.6 \\
\hline 3 & 35.3 & 42.4 & 34.6 & 15.4 \\
\hline 4 & 0.0 & 0.0 & 0.0 & 0.0 \\
\hline 5 & 0.0 & 0.0 & 0.0 & 0.0 \\
\hline
\end{tabular}


ciamientos mayores en la siembra del aguaje evitarían también la competencia por la luz entre plantas.

En el caso de la forma de copa, la mayor cantidad de individuos se encuentra en las categorías buena y aceptable; sin embargo, los porcentajes varían a diferentes distanciamientos de siembra. Mientras que solo el 57.6\% de los individuos tuvo buena forma de copa con distanciamientos mayores de siembra, el $84.6 \%$ de los individuos se ubican en esta categoría a menor distanciamiento de siembra (Tabla 4).

En conclusión, las densidades de siembra en la plantación influyeron sobre el crecimiento en altura, sin llegar a ser estadísticamente significativas. Así mismo, el porcentaje del número de individuos adultos fértiles aumenta con la edad de la plantación, siendo superior en la subparcela con menor densidad de siembra, alcanzando un $50 \%$ a los 12 años de instalada la plantación.

A pesar de no existir diferencias del porcentaje de individuos en las diferentes categorías de iluminación de copas entre las dos subparcelas, existe una marcada competencia entre individuos con requerimientos de luz en las categorías buena y aceptable. Se recomienda continuar con la evaluación y analizar el comportamiento futuro de las plantas de aguaje en plantaciones, evaluando cómo la posición de las copas en el estrato vertical y su relación con sus vecinos de igual o mayor tamaño inciden en los procesos de floración y principalmente en la producción y rendimiento de frutos.

\section{Literatura citada}

Alencar J. 1994. Fenología de cinco especies arbóreas tropicales de Sapotaceae correlacionada a variáveis climáticas na Reserva Ducke, Manaus. Manaus-Brazil. Acta Amazonica 24(3/4):161-182. http://dx.doi. org/10.1590/1809-43921994243182

Baluarte J.R. 2012. Modelización del crecimiento de quince especies forestales comerciales del bosque aluvial inundable de la Amazonía Peruana. Tesis Doctoral, Escola Politécnica superior, Universidad de Santiago de Compostela, 231pp. http://hdl.handle.net/10347/6149

Bejarano P. \& R. Piana. 2002. Plan de manejo de los aguajales aledaños al caño Parinari. Junglevagt for Amazonas WWF - AIF/DK. 85pp.

Cardenas-Valencia L. 1986. Estudio ecológico y diagnóstico silvicultural de un bosque de terraza media en la llanura aluvial del Río Nanay, Amazonía Peruana. Tesis de Maestría, Programa Universidad de Costa Rica/CATIE, 133pp.

Claussi A., D. Marmillod, J. Blaser. 1992. Descripción silvicultural de las plantaciones forestales de Jenaro Herrera. Instituto de Investigaciones de la Amazonía Peruana. Iquitos, 334pp.

Dambroth M. \& N. Bassam. 1983. Low input varieties: definition, ecological requirements and selection. Plant and Soil 72(2-3):365-377. https://doi.org/10.1007/ BF02181974

Del Castillo D., Otárola E. \& L. Freitas. 2006. La maravillosa palmera de la Amazonía Peruana. Instituto de Investigaciones de Investigaciones de la Amazonía Peruana. 51pp.
Delgado C., Couturier G., \& K. Mejía. 2007. Mauritia flexuosa (Arecaceae: Calamoideae), an Amazonian palm with cultivation purposes in Peru. Fruits 62: 157-159. https://doi.org/10.1051/fruits:2007011

Díaz J., M. Paredes \& J. Martinez. 2015. Evaluación participativa de la fenología de las palmeras aguaje (Mauritia flexuosa) y ungurahui (Oenocarpus bataua) en la comunidad nativa Tres Islas, Madre de Dios. USAID, ECOLEX, Rainforest Alliance.

Flores S. 1997. Cultivo de frutales nativos amazónicos; manual para el extensionista. Tratado de Cooperación Amazónica. $370 \mathrm{pp}$.

Freitas L. \& H. Flores. 2015. Condición silvicultural de la palmera Mauritia flexuosa L.f. en el ecosistema "aguajal" de Parinari, Loreto, Perú. Folia Amazónica 24(2): 155162. https://doi.org/10.24841/fa.v24i2.73

Freitas L. 2012. Impacto del aprovechamiento en la estructura, producción y valor de uso del aguaje en la Amazonía peruana. Recursos Naturales y Ambiente 67:38-47.

Freitas L., M. Pinedo, C. Linares et al. 2006. Descriptores para el aguaje (Mauritia flexuosa L. f.). IIAP. 33pp.

García A. 2001. Estudio de la demanda de Mauritia flexuosa L.f. en la ciudad de Iquitos. IIAP.

Gauthier L. \& R. Spichiger. 1986. Ritmos de reproducción en el estrato arbóreo del Arboretum Jenaro Herrera (prov. de Requena, depto. de Loreto, Perú); contribución al estudio de la flora y vegetación de la Amazonia peruana X. Candollea 41(1):193-207.

Gonzales V. \& A. Rial. 2011. Las comunidades de morichal en los Llanos Orientales de Venezuela, Colombia y el Delta del Orinoco, in Lasso C.A., A. Rial, C. Matellana, et al. (Ed.), Biodiversidad de la cuenca del Orinoco. Instituto de recursos Biológicos Alexander Von Humboldt, WWF Colombia, Fundación Omacha, Fundación la Salle de Ciencias Naturales e Instituto de Estudios de la Orinoquía (Universidad Nacional de Colombia). Bogotá D.F. Colombia, pp. 116-139.

Gonzales A., Jarama A. \& G. Chuquival. 2006. Colección y evaluación de germoplasma (Mauritia flexuosa L.f.) aguaje en la Amazonía Peruana. Folia Amazónica 15(1-2):1928. https://doi.org/10.24841/fa.v15i1-2.222

Guzmán-Castillo, W. 2007. Valor económico del manejo sostenible de los ecosistemas de aguaje (Mauritia flexuosa), in International Congress on Development, Environment and Natural Resources: Multi-level and Multi-scale Sustainability, vol. 3, edited by J. Feyen et al., pp. 15131521, Univ. Mayor San Simón, Cochabamba, Bolivia.

Henderson A. 1995. The palms of the Amazon. Oxford University Press. 362pp.

Horn C.M., M.P. Gilmore \& B.A. Endress. 2012. Ecological and socio-economic factors influencing aguaje (Mauritia flexuosa) resource management in two indigenous communities in the Peruvian Amazon. Forest Ecology and Management 267:93-103. https://doi. org/10.1016/j.foreco.2011.11.040

Hutchinson I. 1993. Puntos de partida y muestreo diagnóstico para la silvicultura de bosques naturales del trópico húmedo. Turrialba, CR, CATIE. 32pp. (Serie Técnica. Informe técnico no. 204. Colección Silvicultura y Manejo de Bosques Naturales N. 7).

IIAP-INCAGRO (Instituto de Investigaciones de la Amazonía Peruana - Innovación y Competitividad para el Agro Peruano). 2010. Programa de mejoramiento genético del aguaje, Periodo 2010-2020. IIAP-INCAGRO, 49pp. 
Manzi M. \& O.T. Coomes. 2009. Managing Amazonian palms for community use: a case of aguaje palm (Mauritia flexuosa) in Peru. Forest Ecology and Management 257:510517. https://doi.org/10.1016/j.foreco.2008.09.038

Rathcke B. \& E. Lacey. 1985. Phenological Patterns of terrestrial plants. Annual Annual Review of Ecology and Systematics 16:179-214. https://doi.org/10.1146/annurev.es.16.110185.001143

Rojas R., G. Ruiz, P. Ramírez et al. 2001. Comercialización de masa y «fruto verde» de Aguaje (Mauritia Flexuosa L.f.) en Iquitos (Perú). Folia Amazónica 12(1-2):15-38. DOI https://doi.org/10.24841/fa.v12i1-2.123

Rojas R. 2000. Estado del Conocimiento Sobre El Aguaje (Mauritia flexuosa L.f.). Programa de Ecosistemas Terrestres. IIAP. 74pp.

Santos, L.M.P. 2005. Nutritional and ecological aspects of buriti or aguaje (Mauritia flexuosa Linnaeus filius): a carotene-rich palm fruit from Latin America. Ecology of Food and Nutrition 44:(1-14). https://doi. org/10.1080/03670240500253369.

Synnott T.J. 1991. Manual de procedimientos de parcelas permanentes para bosque húmedo tropical. Trad. J Valerio. CR, Instituto Tecnológico de Costa Rica. 103pp.

Urrego L.E. 1987. Estudio preliminar de la fenología de la canangucha (Mauritia flexuosa L.f.). Colombia Amazónica 2(2):57-81.

Villachica H. 1996. Frutales y hortalizas promisorias de la Amazonía. SPTTCA, $\mathrm{N}^{\circ} 44.367 \mathrm{pp}$.

Villasana R. \& A. Suárez. 1997. Estudio fenológico de dieciséis especies forestales presentes en la Reserva Forestal Imataca Estado Bolívar-Venezuela. Revista Forestal Venezolana 41(1):13-21.

\section{Agradecimientos:}

Los autores expresan sus agradecimientos a los Sres. Arístides Vásques Vásques y Julio Irarica Pacaya, técnicos de campo del Centro de Investigaciones Jenaro Herrera ( $\mathrm{CIJH})$. A Cesar Sabogal, Euridice Honorio y Fidel Chiriboga-Arroyo, por sus aportes y revisión del documento.

\section{Conflicto de intereses:}

Los autores no incurren en conflictos de intereses.

Rol de los autores:

LFA desarrollo el proyecto, participo en el trabajo de campo. RZG realizo el analisis de datos. RBL participo en el trabajo de campo y registro de informacion. LFA, RZG, RBL y DdCT elaboraron el manuscrito.

\section{Fuentes de financiamiento:}

Recursos Ordinarios del Sector Público a través de Programa Presupuestal 144 Conservación y uso sostenible de ecosistemas para la provisión de servicios eco sistémicos, ejecutado por el Instituto de Investigaciones de la Amazonía Peruana.

\section{Aspectos éticos / legales:}

Este trabajo no incurrió en ningún problema ético ni legal. 